\title{
Wearable, Small, and Robust: the Circular Quarter-Mode Textile Antenna
}

\author{
Sam Agneessens, Student Member, IEEE, Sam Lemey, Thomas Vervust, Hendrik Rogier, Senior \\ Member, IEEE
}

\begin{abstract}
A miniaturized wearable antenna, entirely implemented in textile materials, is proposed that relies on a Quarter-Mode Substrate Integrated Waveguide topology. The design combines compact dimensions with high Body-Antenna Isolation, making it excellently suited for off-body communication in wearable electronics/smart textile applications. The fabricated antenna achieves stable on-body performance. A measured on-body impedance matching bandwidth of $5.1 \%$ is obtained, versus $4.8 \%$ in free space. The antenna gain equals 3.8 $\mathrm{dBi}$ in the on-body and $4.2 \mathrm{dBi}$ for the free space scenario. High radiation efficiency, measured to be $81 \%$ in free space, is combined with a low calculated Specific Absorption Rate of 0.45 $\mathrm{mW} / \mathrm{g}$, averaged over $1 \mathrm{~g}$ of tissue, with $500 \mathrm{~mW}$ input power.
\end{abstract}

Index Terms-Antennas, Wearable antennas, Textile antennas, Substrate Integrated Waveguide antennas, Wearable electronics, Quarter-Mode Substrate Integrated Waveguide, Antenna miniaturization

\section{INTRODUCTION: SMART TEXTILES AND WEARABLE ANTENNAS}

Reduction of society's medical cost by remote telemetry, safer, more efficient and faster rescue operations, and real life monitoring of athletic performance parameters are just a few applications that illustrate the potential of smart wearable systems [1]. As a result, the area attracts enormous attention from industry, research, and design groups. The no less than stellar rise of smart phone use in everyday life has demonstrated that people are willing to embrace new technology if it combines ease of use, helpful functionality, and attention to design and aesthetics.

This is why textile antenna designers should pay sufficient attention to smooth and comfortable integration of electronics into garments. Additionally, smart textiles are, in essence, portable wireless systems powered by batteries with limited lifetime and require a highly efficient antenna to achieve optimal performance, in terms of battery lifetime, wireless range, etc. The design of a wearable antenna for on-body application should take these two conflicting requirements

This paper is submitted for review on the 28th August 2014. This work was supported in part by the Inter-University Attraction Poles Program initiated by the Belgian Science Policy Office.

S. Agneessens, S. Lemey, and H. Rogier are with the Department of Information Technology, Ghent University/iMinds, Ghent, B-9000, Belgium (e-mail: sam.agneessens@intec.ugent.be).

Thomas Vervust is with the Department of Electronics and information systems, Ghent University/IMEC. (smooth integration/small dimensions and top performance) into account from the early stages of the design process.

For flexible textile antennas the designer must anticipate mechanical influences (bending, crumpling, and compression), unwanted absorption of radiation by the body (reduced antenna efficiency, potential health threats when Specific Absorption Rate (SAR) limits are violated), frequency detuning, etc. High body-antenna Isolation is needed to avoid these problems and a dedicated antenna design is indispensable to achieve the wearable systems' full potential.

Many textile antenna designs have been reported in literature. They are divided into two groups based on their application: on-body [2, 3], vs. off-body communication. The former group focuses on data transmission along the body between user-worn nodes. The latter type transmits from a body worn device to an off-body device. Most off-body communication antennas rely on microstrip or coplanar waveguide (CPW) topologies: basic patch antennas [4], CPW patches over an Electromagnetic Bandgap (EBG) substrate [5], UWB designs [6], among others. These designs use a ground plane or EBG substrate, with dimensions significantly larger than the antenna itself to shield the radiating element from the human body and achieve good body-antenna isolation.

Recently, antennas based on Substrate Integrate Waveguides (SIW) have been implemented in textile materials. These cavity-backed designs rely on metalized eyelets [7] or embroidered conductive thread [8] to realize the vertical walls of vias defining the cavity. They are excellent components for wearable applications and offer the designer a lot of flexibility, such as wideband [9] or multiband [10] operation. Also of interest are their miniaturization possibilities, by exploiting symmetry of the field distributions of the resonant modes. A half-mode SIW (HMSIW) textile antenna was already proposed [10]. On rigid substrates, the mode symmetry of a square rectangular resonator has recently been used to achieve quarter mode operation [11].

In this letter, advantage is taken of the mode symmetries in a circular waveguide resonator to achieve a very robust on-body antenna with small dimensions. The antenna exhibits a circular QMSIW topology with additional slots to further reduce the size, in the meanwhile improving its integration into garments.

\section{Wearable Antenna Design Methodology}

\section{A. Design goals and considerations}

The proposed miniaturized wearable antenna is designed 
specifically for off-body communication, aiming at excellent, robust on-body performance by high body-antenna isolation. As operating band, the $2.4 \mathrm{GHz}$ ISM band is selected, since this frequency range is used by popular radio protocols, such as Wi-Fi, Bluetooth, and ZigBee, making the design suitable for a plethora of applications. The compactness and its fabrication based on materials, commonly found in garments (which may be specific to the target application), and on electro textiles, realizing the conductive planes, contribute to the wearability of the design.

The antenna specifications in terms of EM performance are strongly influenced by the on-body environment. Robust operation is vital. A reflection coefficient, $\Gamma$, lower than $-10 \mathrm{~dB}$ and an efficiency of more than $60 \%$, which implies that the link budget would decrease by less than $2 \mathrm{~dB}$ due to antenna losses, are imposed in the entire ISM band. As the distance between body and antenna cannot be fixed with absolute certainty, these specs should be satisfied both for deployment in free space and on the human body. The SAR value should also remain below the limit of $1.6 \mathrm{~mW} / \mathrm{g}$ averaged over $1 \mathrm{~g}$ of tissue, given the IEEE C95.1 standard.

\section{B. Design evolution: miniaturization of the wearable antenna topology}

To meet these performance requirements, an antenna topology based on SIW technology implemented in textile materials is adopted. SIW designs combine easy fabrication of planar microwave components with the performance of conventional bulky waveguides. Rows of conducting vias are used to connect two conducting sides of a substrate, effectively forming a waveguide. This approach results in good isolation between antenna and body. Furthermore, the symmetry of mode distributions can be used to reduce the dimensions while maintaining the performance. This technique is used twice to achieve excellent on-body performance and small dimensions. The design evolution consists of three simple steps shown in Fig. 1. A circular resonating cavity, operating at its fundamental mode, is the start point. The dimensions are determined by the well-known analytical formulas for circular waveguides and adjusted based on the SIW equivalent waveguide width [12], taking into account via spacing guidelines for SIW to minimize radiation leakage.

In a first step, the symmetry of the magnetic field is exploited to reduce the dimensions of the cavity. A virtual magnetic wall A can be drawn along the horizontal symmetry plane of the cavity. Cutting away the cavity below the line, while keeping a ground plane extension of length $g n d_{e x t}$, results in a semicircular cavity resonating in half-mode operation. Another symmetry plane can be drawn along virtual magnetic wall B. Applying the same procedure, the dimensions of the antenna can be reduced again by almost half.

Now, the cavity operates at quarter-mode and radiation leakage through the open side wall results in efficient antenna operation. The antenna radiates in a hemisphere with direction of largest gain in the z-direction. This minimizes radiation towards the body (the $-\mathrm{z}$ direction), leading to high bodyantenna isolation and, hence, robust on-body performance. In a final step, two slots are added to lengthen the current path on the top patch, which reduces the size even more.

The structure is fed by a coaxial probe placed on the $\varphi=45^{\circ}$ axis. The current loop, formed by the probe and the metalized eyelets, effectively couples to the magnetic field of the resonant cavity mode. Fig. 2 shows the distribution of the electric and magnetic field at $2.45 \mathrm{GHz}$ in the antenna cavity. The symmetrical geometry of the antenna w.r.t. $\varphi=45^{\circ}$, and the equal contributions of the leaking cavity walls and slots on each side result in a linearly polarized antenna with its copolarization and cross-polarization axis along $\varphi=45^{\circ}$ and $\varphi=135^{\circ}$, respectively.

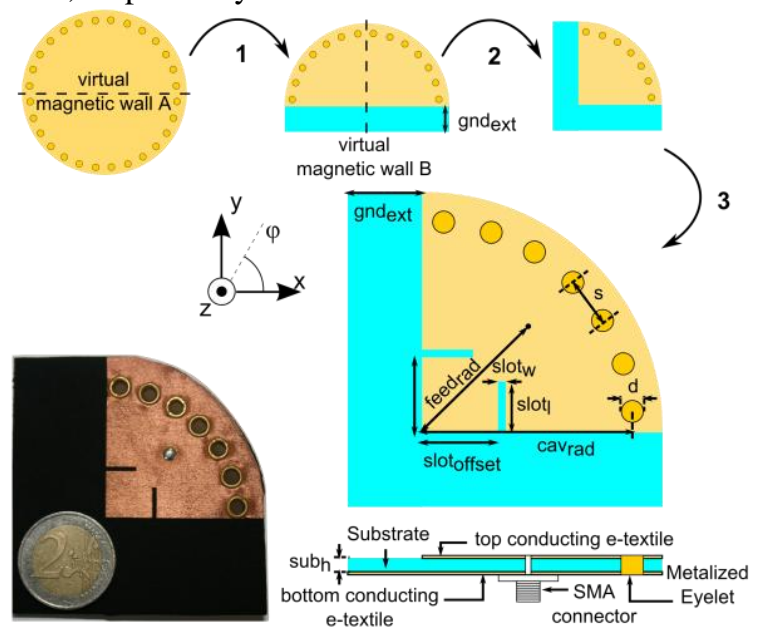

Fig. 1. Design evolution: the symmetry of the first resonant mode is exploited in the first two steps, in addition to current displacing slots added in the final step, to significantly reduce the dimensions while maintaining high body-antenna isolation. Inset: Photograph of fabricated antenna

Dimensions of final design (in $\mathrm{mm}$ ): $\operatorname{cav}_{\mathrm{rad}}=34.9, \operatorname{slot}_{\mathrm{l}}=8, \operatorname{slot}_{\mathrm{w}}=1$, slot $_{\text {offset }}=12.9$, feed $_{\text {rad }}=17.4$, gnd $_{\text {ext }}=25, \mathrm{~d}=4, \mathrm{~s}=8$.

\section{Design parameters and optimization}

This 3-step miniaturization process leads to an elegant smallsize antenna with straightforward design parameters.

The substrate height $s u b_{h}$ influences the radiation leakage from the cavity and the impedance bandwidth (BW). A larger height results in wider BW, higher radiation efficiency, and reduced resonance frequency, but at the cost of a more bulky antenna. The substrate's dielectric properties $\left(\varepsilon_{r}\right.$ and $\left.\tan \delta\right)$ influence the performance identical to regular patch antennas. $c a v_{\text {rad }}$ is the most important parameter in determining the operating frequency with an inverse relation between cavity radius and resonant frequency. The slots are identical in offset and size and their main function is antenna miniaturization. Increasing their dimension reduces the operating frequency and BW simultaneously. Slot offset is useful for fine-tuning the frequency. The final slot length of $8 \mathrm{~mm}$ reduces the resonance frequency by $112 \mathrm{MHz}$ while still meeting the $\mathrm{BW}$ requirements. The position of the feed, feed $d_{r a d}$, is important for matching. Optimal feed position depends on the desired input impedance and is ideally placed near the cavity wall.

The size of the ground plane is an important design parameter w.r.t the on-body performance. A large ground plane leads to large antenna dimensions, whereas a too small ground plane results in poor on-body performance: impedance matching 
deteriorates, the resonance frequency shifts, efficiency drops drastically, and body absorption (SAR level) increases. To determine the optimal size, the influence of gnd $_{\mathrm{ext}}$ on the onbody performance is investigated by sweeping the parameter for an antenna in the vicinity of a human body (distance $2 \mathrm{~mm}$ ). The radiation pattern and impedance matching for each swept value are evaluated. Smaller values of gnd $_{\text {ext }}$ show limited, but clearly noticeable, influence on the impedance matching. A stable characteristic is achieved when the $\mathrm{gnd}_{\mathrm{ext}}$ becomes larger than $0.15 \lambda$. The effect on the radiation pattern and efficiency is more significant. Absence of a ground plane $\left(\mathrm{gnd}_{\mathrm{ext}}=0\right)$ leads to a strongly deteriorated gain pattern and low on-body radiation efficiency (38\%). Increasing the size of the ground plane immediately results in significantly better performance. Once the ground plane is large enough $(>0.15 \lambda)$ the radiation efficiency remains stable. The presence of the body still deforms the radiation pattern slightly. By increasing $\mathrm{gnd}_{\mathrm{ext}}$ the gain pattern will more closely resemble the free space one, as a consequence of the increased body-antenna isolation. Since an identical on-body and free space radiation pattern is not a design requirement for our wearable antenna, a gnd $_{\text {ext }}$ value is adopted that provides an excellent trade-off between sufficiency body-antenna isolation and size.

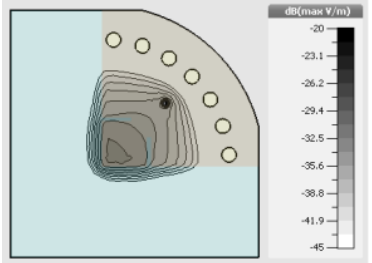

(a)

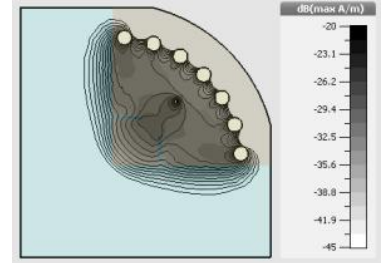

(b)
Fig. 2. (a) E-Field and (b) H-field distribution in the QMSIW antenna at $2.45 \mathrm{GHz}$

The relation between via spacing and diameter is assumed to be fixed to $\mathrm{s}=2 \mathrm{~d}$. If $\mathrm{d}$ is reduced, the equivalent waveguide width will also decrease, leading to an overall smaller antenna. Optimization of the antenna is carried out with the CST Microwave Studio EM field simulator. Its final layout and dimensions are shown in the caption of Fig. 1.

\section{Textile antenna fabrication}

Since the aim is for comfortable and unobtrusive integration into garments, the antenna is fabricated with off-the-shelf (electro) textile materials and standard apparel manufacturing techniques. The conductive planes on both sides of the substrate are realized with a conductive e-textile copper-plated polyester taffeta fabric with thickness $80 \mu \mathrm{m}$ and surface resistivity $0.18 \Omega / \mathrm{sq}$ at $2.45 \mathrm{GHz}$. These sheets are patterned by laser cutting with a picosecond pulsed laser with a spot size of $20 \mu \mathrm{m}$, resulting in very accurate fabrication dimensions. A thermally activated adhesive sheet is applied to attach these etextiles to the antenna substrate, which is a flexible closed-cell expanded rubber protective foam, commonly found in firefighter gear, (thickness $\mathrm{h}=3.7 \mathrm{~mm}, \varepsilon_{\mathrm{r}}=1.495, \tan \delta=$ 0.016). To implement the metalized vias connecting both ground planes, brass eyelets are punched through this assembly by means of a hand press.

\section{Wearable ANTENNA Design Validation}

To validate the design, the simulation results are compared with measurements performed in an anechoic. For antenna deployed on a human body, the design is positioned centered on the chest of a person, wearing a shirt, with a body mass index (BMI) of 26.8 .

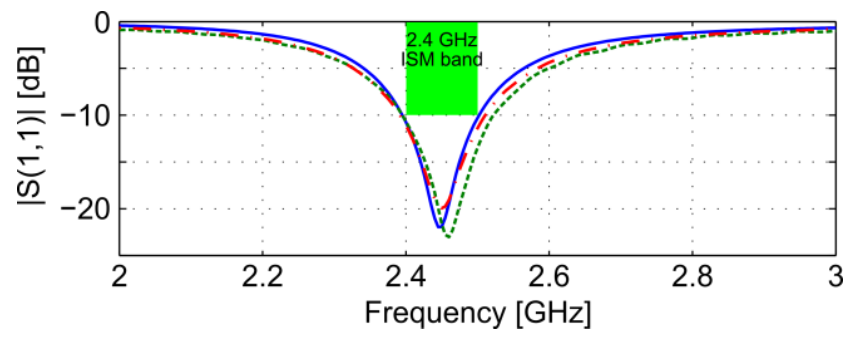

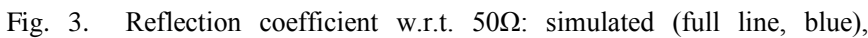
measured (dot-dashed line, red), and on-body measured (dotted line, green). There is good matching in the complete $2.4 \mathrm{GHz}$ ISM band, both in free space and on-body.

In Fig. 3, the simulated and measured reflection coefficient, w.r.t. $50 \Omega$, are shown, evaluated both in free space and after deployment on the body. Free space simulation and measurement agree very well. The antenna's measured fractional bandwidth w.r.t $-10 \mathrm{~dB}$ is $4.8 \%(117 \mathrm{MHz})$, which is close to the simulated value of $4.4 \%(107 \mathrm{MHz})$. As for the on-body measurement, a stable resonance frequency, without frequency shift, and stable shape of the reflection curve are observed. Hence, the input impedance of the proposed antenna remains stable in proximity of the human body. The bandwidth has slightly increased to $5.1 \%(128 \mathrm{MHz})$, due to additional losses caused by the presence of the human body. Cross sections of the radiation pattern of the antenna in the Eand H-plane, at the center frequency of $2.45 \mathrm{GHz}$, are shown in Figs. 4.a and 4.b. The measured and simulated free-space results agree very well, both in terms of performance and pattern. A maximum simulated and measured gain of $4.4 \mathrm{dBi}$ and $4.2 \mathrm{dBi}$, respectively, is obtained and a simulated radiation efficiency of $76 \%$ versus a measured value of $81 \%$. The measured on-body gain in the H-plane $\left(\varphi=135^{\circ}\right)$ agrees very well with the measured free space result, showing the expected symmetrical pattern. For the E-plane $\left(\varphi=45^{\circ}\right)$, the measured free space and on-body gains are also very comparable, except at low elevation angles, $\theta$, between $-90^{\circ}$ and $-45^{\circ}$, where the pattern is noticeably distorted. This is due to radiation absorbed by the body. This effect can be reduced by increasing the ground extension. Maximum on-body measured gain equals $3.8 \mathrm{dBi}$. Both plots clearly show that radiation in the negative $\mathrm{z}$-direction (towards the body) is significantly attenuated by the body. Radiation in this direction was already very small owing to the dedicated on-body design so only little power is lost by undesirable body absorption. Fig. 4 also included the measured crosspolarization patterns of the antenna in free space and for the on-body scenario. In free space, the presence of the human 
body does not significantly increase the cross-polarization levels and the antenna remains linearly polarized in the onbody scenario.

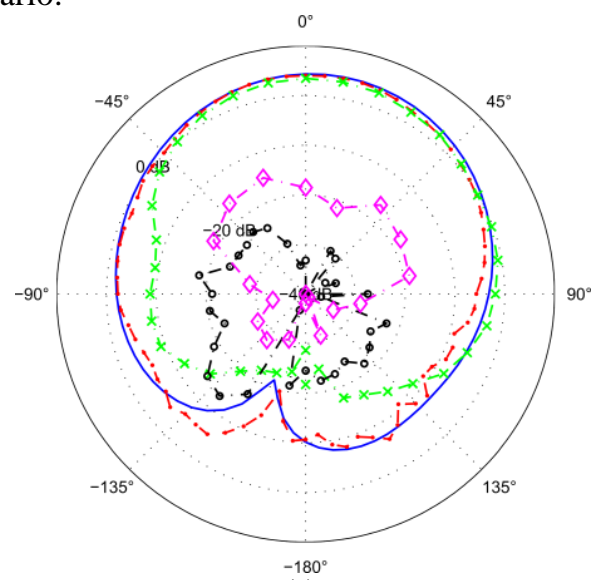

(a)

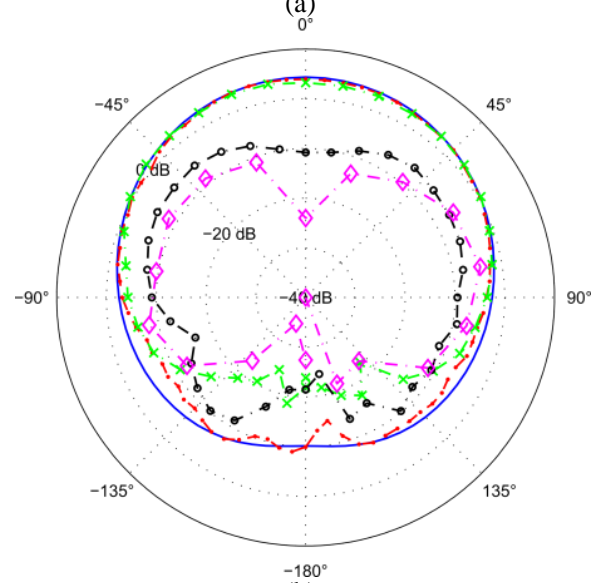

(b)

Fig. 4. Antenna radiation pattern in the (a) E-plane $\left(\varphi=45^{\circ}\right)$ and (b) H-plane $\left(\varphi=135^{\circ}\right)$ : free space, simulated (full line, blue) and measured (dot dashed red line); on-body measurement (dotted green line, $\mathrm{x}$-marker); crosspolarization for free space (black, circle) and on-body (magenta, diamond) scenario.

The Specific Absorption Rate for the antenna placed at $2 \mathrm{~mm}$ distance from the human body was calculated. The model used is the three layer human body model [13]. From these simulations, a $0.45 \mathrm{~mW} / \mathrm{g}$ averaged over $1 \mathrm{~g}$ of tissue with $500 \mathrm{~mW}$ input power is achieved. This value is well below the SAR limit. Simulations relying on a human body model result in an estimated on-body radiation efficiency of $60 \%$, corresponding to a reduction of only $1 \mathrm{~dB}$ in radiation efficiency due to the presence of the human body. This result compares very favorably to other wearable antenna designs in recent literature.

\section{CONCLUSIONS}

A new dedicated wearable antenna topology gave rise to a highly integrable wearable design for off-body communication with excellent free space and on-body performance. The design leverages substrate integrated waveguide technology implemented on textiles with quarter-mode miniaturization techniques to achieve a compact dimensions, wearability, and high body-antenna isolation. Measurements in free space and on-body scenarios validated the design's potential for wearable off-body communications.

\section{REFERENCES}

[1] S. Park and S. Jayaraman, "Enhancing the quality of life through wearable technology," Engineering in Medicine and Biology Magazine, IEEE, vol. 22, pp. 41-48, 2003.

[2] M. Klemm and G. Troester, "Textile UWB antennas for wireless body area networks," Antennas and Propagation, IEEE Transactions on, vol. 54, pp. 31923197, 2006.

[3] G. Conway and W. Scanlon, "Antennas for Over-BodySurface Communication at $2.45 \mathrm{GHz}$," Antennas and Propagation, IEEE Transactions on, vol. 57, pp. 844$855,2009$.

[4] A. Tronquo, H. Rogier, C. Hertleer and L. Van Langenhove, "Robust planar textile antenna for wireless body LANs operating in $2.45 \mathrm{GHz}$ ISM band," Electronics Letters, vol. 42, no. 3, pp. 142-143, 2006.

[5] S. Zhu and R. Langley, "Dual-Band Wearable Textile Antenna on an EBG Substrate," Antennas and Propagation, IEEE Transactions on, vol. 57, no. 4, pp. 926-935, April 2009.

[6] P. Samal, P. Soh and G. Vandenbosch, "UWB All-Textile Antenna With Full Ground Plane for Off-Body WBAN Communications," Antennas and Propagation, IEEE Transactions on, vol. 62, no. 1, pp. 102-108, Jan 2014.

[7] R. Moro, S. Agneessens, H. Rogier and M. Bozzi, "Wearable textile antenna in substrate integrated waveguide technology," Electronics Letters, vol. 48, no. 16, pp. 985-987, August 2012.

[8] T. Kaufmann and C. Fumeaux, "Wearable Textile HalfMode Substrate-Integrated Cavity Antenna Using Embroidered Vias," Antennas and Wireless Propagation Letters, IEEE, vol. 12, pp. 805-808, 2013.

[9] S. Lemey, F. Declercq and H. Rogier, "Dual-Band Substrate Integrated Waveguide Textile Antenna With Integrated Solar Harvester," Antennas and Wireless Propagation Letters, IEEE, vol. 13, pp. 269-272, 2014.

[10] S. Agneessens and H. Rogier, "Compact Half Diamond Dual-Band Textile HMSIW On-Body Antenna," Antennas and Propagation, IEEE Transactions on, vol. 62, no. 5, pp. 2374-2381, May 2014.

[11] C. Jin, R. Li, A. Alphones and X. Bao, "Quarter-Mode Substrate Integrated Waveguide and Its Application to Antennas Design," Antennas and Propagation, IEEE Transactions on, vol. 61, no. 6, pp. 2921-2928, June 2013.

[12] M. Bozzi, A. Georgiadis and K. Wu, "Review of substrate-integrated waveguide circuits and antennas," Microwaves, Antennas Propagation, IET, vol. 5, no. 8, pp. 909-920, June 2011.

[13] M. L. Scarpello, L. Vallozzi, H. Rogier and D. Vande Ginste, "High-gain textile antenna array system for offbody communication," International Journal of Antennas and Propagation, vol. 2012, 2012. 